\title{
Grid-based seismic modelling at high and low signal-to-noise ratios
}

\section{HD 181420 and HD 175272}

\author{
S. Hekker ${ }^{1}$ and W. H. Ball ${ }^{2}$ \\ 1 Max-Planck-Institut für Sonnensystemforschung, Justus-von-Liebig-Weg 3, 37077 Göttingen, Germany \\ e-mail: hekker@mps.mpg.de \\ 2 Institut für Astrophysik, Georg-August-Universität Göttingen, Friedrich-Hund-Platz 1, 37077 Göttingen, Germany
}

Received 25 November 2013 / Accepted 10 March 2014

\begin{abstract}
Context. Recently, the CoRoT target HD 175272 (F5V), which shows a weak signal of solar-like oscillations, was modelled by a differential asteroseismic analysis (Ozel et al. 2013, A\&A, 558, A79) relative to a seismically similar star, HD 181420 (F2V), for which there is a clear signature of solar-like oscillations. The results provided by Ozel et al. indicate the possibility of HD 175272 having subsolar mass, while being of the order of $1000 \mathrm{~K}$ hotter than the Sun. This seems unphysical - standard stellar evolution theory generally does not predict solar-metallicity stars of subsolar mass to be hotter than about $6000 \mathrm{~K}$ - and calls for a reanalysis of this star.

Aims. We aim to compare the performance of differential asteroseismic analysis with that of grid-based modelling.

Methods. We use two sets of stellar model grids and two grid-fitting methods to model HD 175272 and HD 181420 using their effective temperatures, metallicities, large frequency separations $(\Delta v)$, and frequencies of maximum oscillation power $\left(v_{\max }\right)$ as observational constraints.

Results. We find that we are able to model both stars with parameters that are both mutually compatible and comparable with other modelling efforts. Hence, with modest spectroscopic and asteroseismic inputs, we obtain reasonable estimates of stellar parameters. In the case of HD 175272, the uncertainties of the stellar parameters from our grid-based modelling are smaller, and hence more physical, than those reported in the differential analysis. For both stars, the models have significantly lower values of $v_{\max }$ than the reported observed values. Furthermore, when using the asymptotic large frequency separation as opposed to the scaling relation to compute $\Delta v$, we find that our modelling results are significantly more self-consistent when $v_{\max }$ is ignored.

Conclusions. Grid-based modelling is a useful tool even in cases of weak solar-like oscillations. It provides more precise and more realistic results than obtained with differential seismology. The difference in the observed and modelled values of $v_{\max }$ indicates that the four observational constraints are not fully consistent with the stellar models used here, with $v_{\max }$ most likely to be the inconsistent constraint for these two stars.
\end{abstract}

Key words. asteroseismology - stars: individual: HD 175272 - stars: individual: HD 181420

\section{Introduction}

The CoRoT (Baglin et al. 2006; Auvergne et al. 2009) and Kepler (Borucki et al. 2008, 2010) missions have provided a wealth of high-quality, nearly-uninterrupted photometric time series, which are useful for investigations of stellar oscillations. From stellar oscillations in main-sequence stars, subgiants, and red giants it is possible to accurately derive stellar parameters such as mass, radius, mean density, and surface gravity, as well as more detailed knowledge about the internal stellar structure. For example, it has been possible to measure: whether helium is burning in the cores of red giants (Beck et al. 2011; Bedding et al. 2011; Mosser et al. 2011); how much (differential) rotation takes place (Beck et al. 2012; Deheuvels et al. 2012; Mosser et al. 2012); the inclination angle and obliquity between the spin axes of a star and its companions (Chaplin et al. 2013; Huber et al. 2013); the location of the second helium ionization zone (Miglio et al. 2010; Mazumdar et al. 2012, 2014).

Solar-like oscillations - oscillations stochastically excited in the turbulent outer convective zones of low-mass main-sequence stars, subgiants, and red giants - can be analysed to various levels of detail. Accurate measures of individual frequencies are important to study the internal structures of stars. However, it is not always possible to measure individual oscillation frequencies owing, for example, to a low signal-to-noise ratio of the oscillations. In such cases one can use so-called global oscillation parameters: the frequency separation between modes of the same degree and consecutive (acoustic) radial order $\Delta v$, which is roughly proportional to the square root of the mean density of the star; and the frequency of maximum oscillation power $v_{\max }$, which scales with the surface gravity $g$ and square root of the effective temperature $T_{\text {eff }}$. These two parameters can be used to estimate the stellar mass and radius, when combined with effective temperature using well-known scaling relations (Kjeldsen \& Bedding 1995).

The scaling relations are useful but should always be used with their limitations in mind. Firstly, they can be derived by assuming homology in stellar structures. This is clearly an approximation because structural features like the depths of convection zones and the presence (or absence) of convective cores change with mass, metallicity, and age. Comparisons with independently-derived radii and surface gravities have shown 
Table 1. Observed parameters of HD 181420 (F2V) and HD 175272 (F5V) taken from Ozel et al. (2013).

\begin{tabular}{lcc}
\hline \hline & HD 181420 & HD 175262 \\
\hline$\Delta v / \mu \mathrm{Hz}$ & $75.20 \pm 0.04$ & $74.9 \pm 0.4$ \\
$v_{\max } / \mu \mathrm{Hz}$ & $1610 \pm 10$ & $1600 \pm 30$ \\
$T_{\text {eff }} / \mathrm{K}$ & $6580 \pm 100$ & $6675 \pm 120$ \\
{$[\mathrm{Fe} / \mathrm{H}]$} & $-0.05 \pm 0.06$ & $+0.08 \pm 0.11$ \\
\hline
\end{tabular}

that the approximation is reasonably accurate for a wide range of stars, although with an increase in uncertainty for stars with different internal stellar structures compared to Sun-like stars (e.g. White et al. 2011; Huber et al. 2012; Silva Aguirre et al. 2012; Miglio et al. 2012). Secondly, the $\Delta v$ scaling relation is derived in the asymptotic regime where the radial order $n$ is much larger than the degree $l$, i.e. $n \gg l$. This is usually true for oscillations of stars on the main sequence, but not necessarily for more evolved stars. Corrections to account for the nonasymptotic regime are currently being discussed (Mosser et al. 2013; Hekker et al. 2013a).

To constrain stellar age and composition, one can compare the observed global oscillation parameters with those of a large number of stellar models. This is often referred to as grid-based modelling (e.g. Basu et al. 2010; Gai et al. 2011). It has found wide application mostly in the analysis of large sets of data (e.g. Chaplin et al. 2014; Hekker et al. 2013b), but also of stars with weak signatures of oscillation (e.g. Barclay et al. 2013).

Recently, Ozel et al. (2013) proposed to derive stellar parameters for a star with a weak oscillation signal by computing linear differences with respect to the stellar parameters in a second star with a strong oscillation signal and similar observable parameters (e.g. $\left.\Delta v, v_{\max }, T_{\text {eff }},[\mathrm{Fe} / \mathrm{H}]\right)$. Ozel et al. (2013) referred to this method as differential seismology and applied it to the seismically similar stars HD 175272 (F5V) and HD 181420 (F2V) (Bruntt 2009; Barban et al. 2009; Huber et al. 2012) using CESAM models. HD 175272 was observed by CoRoT for 27 days and shows oscillations at a low signal-to-noise ratio. Though the individual oscillation frequencies cannot be identified for HD 175272, global seismic parameters can be determined and were found to be similar to the parameters of the well-studied star HD 181420. This star was therefore used as a reference to derive the parameters of HD 175272.

In this work we present grid-based modelling for both HD 175272 and HD 181420, using two different sets of stellar models and two different grid-modelling methods. The observational constraints for HD 181420 and HD 175272 were taken from Ozel et al. (2013) and are listed in Table 1. We compare our results with the results from differential asteroseismology.

\section{Grid-based modelling}

\subsection{Stellar model grids}

Our grid-based modelling uses two stellar model grids. The first is the canonical BASTI grid $^{1}$ (Pietrinferni et al. 2004), which spans masses from $0.5 M_{\odot}$ to $3.5 M_{\odot}$ in steps of $0.05 M_{\odot}$ and metallicities of $Z=0.0001,0.0003,0.0006,0.001,0.002$, $0.004,0.008,0.01,0.0198,0.03$ or 0.04 . (The corresponding helium abundances are $Y=0.245,0.245,0.246,0.246,0.248$, $0.251,0.256,0.259,0.2734,0.288$, and 0.303.) The BASTI grid includes models from the zero-age main sequence all the

\footnotetext{
1 http://albione.oa-teramo.inaf.it/
}

way to the asymptotic giant branch phase. The models were computed using an updated version of the code described by Cassisi \& Salaris (1997) and Salaris \& Cassisi (1998). Opacities were taken from the OPAL tables (Iglesias \& Rogers 1996) and Ferguson et al. (2005) for $\log T>4$ and $\log T \leq 4$, respectively. The solar abundance pattern and solar metallicity were taken from Grevesse \& Noels (1993) and no diffusion was included. Nuclear reaction rates are drawn from the NACRE database (Angulo et al. 1999), with the particular rate for ${ }^{12} \mathrm{C}(\alpha, \gamma){ }^{16} \mathrm{O}$ from the calculation by Kunz et al. (2002). The models used a contemporary version of the FreeEOS equation of state $^{2}$ (Irwin 2012) and the atmosphere model by Krishna Swamy (1966). Convection is described by the formulation of mixing-length theory (Böhm-Vitense 1958) given by Cox (1968), with the mixinglength parameter fixed at a solar-calibrated value. Finally, mass loss is included according to the empirical relation by Reimers (1975) with the efficiency parameter $\eta$ taking the widely-used value of 0.4 .

The second grid was constructed using $\mathrm{MESA}^{3}$ (Paxton et al. 2011, 2013, revision 5527) and covered the same selection of initial metallicities and helium abundances as the BASTI grid but with masses from $0.8 M_{\odot}$ to $1.8 M_{\odot}$ in steps of $0.01 M_{\odot}$. Models were evolved from the pre-main-sequence up to a maximum age of $15 \mathrm{Gyr}$ or a maximum radius of $25 R_{\odot}$, well up the red-giant branch, with a maximum timestep of 50 Myr. Opacities are drawn from the OPAL tables (Iglesias \& Rogers 1996) and Ferguson et al. (2005) at high and low temperatures, respectively, and are smoothly blended over the entire region where the tables overlap $(3.75 \leq \log T \leq 4.50)$. The models use a standard Eddington grey atmosphere and convective processes are described by mixing-length theory according to Henyey et al. (1965) with mixing-length parameter $\alpha=1.9$. All models use a scaled solar composition according to the values given by Grevesse \& Sauval (1998) without any chemical diffusion. Nuclear reaction rates are taken from Caughlan \& Fowler (1988) or the NACRE collaboration (Angulo et al. 1999), with preference given to the latter when available. Unlike the BASTI grid, no mass loss was included. All other options took their default values described in the MESA instrument papers (Paxton et al. 2011, 2013).

\subsection{Asteroseismic parameters}

Given the mass $M$, radius $R$, and effective temperature $T_{\text {eff }}$ of a stellar model, $v_{\max }$ and $\Delta v$ can be estimated using the commonlyused scaling relations (Kjeldsen \& Bedding 1995):

$\frac{v_{\max }}{v_{\max , \odot}}=\frac{M}{M_{\odot}}\left(\frac{R}{R_{\odot}}\right)^{-2}\left(\frac{T_{\mathrm{eff}}}{T_{\mathrm{eff}, \odot}}\right)^{-\frac{1}{2}}$,

and

$\frac{\Delta v}{\Delta v_{\odot}}=\left(\frac{M}{M_{\odot}}\right)^{\frac{1}{2}}\left(\frac{R}{R_{\odot}}\right)^{-\frac{3}{2}}$,

where the subscript $\odot$ denotes solar values, for which we took $v_{\text {max }, \odot}=3090 \pm 30 \mu \mathrm{Hz}, \Delta v_{\odot}=135.1 \pm 0.1 \mu \mathrm{Hz}$, and $T_{\text {eff }, \odot}=$ $5778 \mathrm{~K}$ (Huber et al. 2011). The uncertainties in the solar values are taken into account in the grid-based modelling by adding them quadratically to the uncertainties in the observations (Chaplin et al. 2014). We note here that we did not use the

\footnotetext{
2 http://freeeos.sourceforge.net/

3 http://mesa.sourceforge.net/
} 
Table 2. Grid-based modelling results.

\begin{tabular}{|c|c|c|c|c|c|c|c|c|}
\hline \multirow{3}{*}{$\begin{array}{l}\text { Model grid } \\
\text { method }\end{array}$} & \multicolumn{4}{|c|}{ HD 181420} & \multicolumn{4}{|c|}{ HD 175272} \\
\hline & BASTI & BASTI & $\operatorname{MESA}^{a}$ & $\mathrm{MESA}^{b}$ & BASTI & BASTI & $\operatorname{MESA}^{a}$ & $\mathrm{MESA}^{b}$ \\
\hline & LD & PySEEK & PySEEK & PySEEK & LD & PySEEK & PySEEK & PySEEK \\
\hline$M / M_{\odot}$ & $1.40 \pm 0.04$ & $1.40_{-0.03}^{+0.03}$ & $1.41_{-0.02}^{+0.04}$ & $1.49_{-0.02}^{+0.05}$ & $1.48 \pm 0.08$ & $1.48_{-0.07}^{+0.06}$ & $1.50_{-0.07}^{+0.07}$ & $1.59_{-0.07}^{+0.05}$ \\
\hline$t / \mathrm{Gyr}$ & $1.81_{-0.07}^{+0.07}$ & $1.8_{-0.1}^{+0.5}$ & $2.0_{-0.4}^{+0.2}$ & $1.3_{-0.2}^{+0.2}$ & $1.4_{-0.6}^{+1.0}$ & $1.3_{-0.3}^{+0.5}$ & $1.4_{-0.4}^{+0.4}$ & $1.0_{-0.3}^{+0.4}$ \\
\hline$R / R_{\odot}$ & $1.65 \pm 0.06$ & $1.65_{-0.02}^{+0.01}$ & $1.66_{-0.01}^{+0.02}$ & $1.712_{-0.002}^{+0.002}$ & $1.68 \pm 0.06$ & $1.69_{-0.03}^{+0.02}$ & $1.69_{-0.03}^{+0.03}$ & $1.74_{-0.03}^{+0.01}$ \\
\hline$\Delta v / \mu \mathrm{Hz}$ & $75.24 \pm 0.01$ & $75.26_{-0.16}^{+0.05}$ & $75.20_{-0.03}^{+0.19}$ & $75.16_{-0.01}^{+0.04}$ & $75.1 \pm 0.5$ & $75.1_{-0.4}^{+0.4}$ & $75.1_{-0.4}^{+0.4}$ & $75.1_{-0.4}^{+0.4}$ \\
\hline$\nu_{\max } / \mu \mathrm{Hz}$ & $1483.5 \pm 0.4$ & $1484_{-3}^{+2}$ & $1490_{-1}^{+4}$ & $1449_{-11}^{+30}$ & $1496 \pm 25$ & $1500_{-20}^{+17}$ & $1508_{-24}^{+17}$ & $1484_{-24}^{+27}$ \\
\hline$T_{\text {eff }} / \mathrm{K}$ & $6574_{-14}^{+14}$ & $6583_{-165}^{+7}$ & $6552_{-72}^{+124}$ & $6780_{-82}^{+175}$ & $6662_{-144}^{+147}$ & $6648_{-87}^{+153}$ & $6661_{-116}^{+125}$ & $6810_{-119}^{+116}$ \\
\hline$[\mathrm{Fe} / \mathrm{H}]$ & $-0.25 \pm 0.01$ & $0.06_{-0.09}^{+0.09}$ & $0.06_{-0.09}^{+0.09}$ & $0.06_{-0.09}^{+0.09}$ & $0.07 \pm 0.03$ & $0.22_{-0.18}^{+0.11}$ & $0.20_{-0.18}^{+0.11}$ & $0.25_{-0.15}^{+0.09}$ \\
\hline
\end{tabular}

Notes. ${ }^{(a)} \Delta v$ from scaling relations. ${ }^{(b)} \Delta v$ from asymptotic large frequency spacing $\left(\Delta v_{\text {as }}\right.$, see Eq. (3)).

corrections to the observed and reference $\Delta v$ values proposed by Mosser et al. (2013) as the analysis of a large sample of solarlike stars has shown that this does not have significant impact on the results (Chaplin et al. 2014).

For the MESA grid, we also computed the asymptotic large frequency separation $\Delta v_{\text {as }}$ given by

$\Delta v_{\mathrm{as}}=\left(2 \int_{0}^{R} \frac{\mathrm{d} r}{c(r)}\right)^{-1}$,

where $c$ is the local speed of sound and $r$ the radial co-ordinate inside the star. In other words, the asymptotic large frequency separation is the inverse of the time taken for a sound wave to travel from one side of the star to the other. The MESA grid includes the model values of $\Delta v_{\text {as }}$, so we performed two separate fits: one with $\Delta v$ from the scaling relations and one with $\Delta v$ equal to its asymptotic value. These are marked by superscript $a$ and $b$ in Table 2. When using the asymptotic separation, we did not include the uncertainty in $\Delta v_{\odot}$ in the observational constraints.

\subsection{Model fitting methods}

We used two methods to fit stellar models to observations. The first method is an independent implementation of the likelihood method described by Basu et al. (2010). In short, the likelihood of each model is computed given the values of some chosen set of observed parameters. To obtain a reliable uncertainty for the derived parameters a Monte Carlo analysis is performed, in which the observed values are perturbed within their uncertainties and a new likelihood is determined. The final answer is derived from the centre and width of a Gaussian fit through the total likelihood distribution of 1000 perturbations. We refer to this method as LD (Likelihood Distribution).

The second method is an independent implementation of the SEEK method (Quirion et al. 2010), which we refer to as PySEEK. PySEEK calculates the Gaussian probability of each stellar model given the observations and constructs a probabilityweighted histogram for the desired parameter. The cumulative sum of the histogram is computed and a linear interpolation is used to find where the cumulative sum takes values 0.1585 , 0.5 , and 0.8415 , which correspond to the one-sigma limits of a Gaussian distribution. These values are then taken as the $\pm 1 \sigma$ intervals that are reported. This is the same method that was used for the GOE results reported in Chaplin et al. (2014).
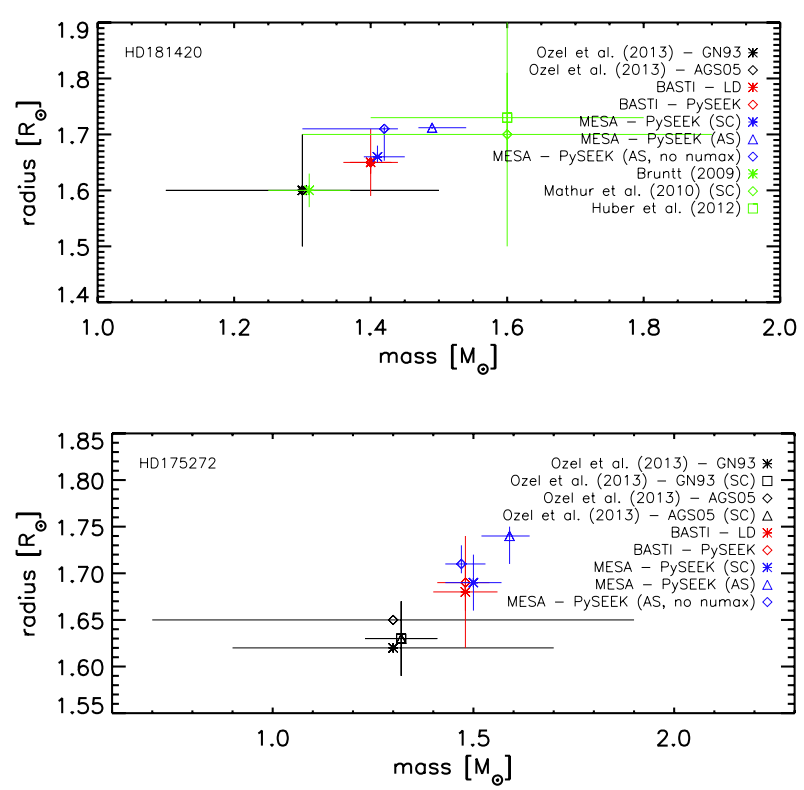

Fig. 1. Radii and masses determined for HD 181420 (top) and HD 175272 (bottom) using the differential seismology using CESAM models (black, Tables 3 and 4), grid-based modelling using BASTI models (red, Table 2), grid-based modelling using MESA models (blue, Tables 2 and 5), and literature values (green, Table 3). See legend for more information.

\section{Results}

We present the results of our grid-based modelling in Table 2. Figure 1 shows fitted values of the masses and radii, along with results from other works. The four sets of results are mutually compatible. When both using the BASTI models, the two different grid search methods agree very well, as should be expected. The results derived using scaling relations and the MESA grid also agree with both sets of BASTI-based results. When fitting $\Delta v$ with the asymptotic large separation (Eq. (3)), the results for the MESA grid differ and consistently show larger masses, radii, and effective temperatures. The differences in the masses and radii are consistent with the differences noted by White et al. (2011) in the sense that the models that are fitted with the asymptotic separation have smaller mean densities. Though the parameters for HD 175272 still agree reasonably with the results derived with scaling relations, the parameters for HD 181420 
Table 3. Parameters derived for HD 181420 in the literature.

\begin{tabular}{lcccccc}
\hline \hline Reference & Bruntt (2009) & Mathur et al. (2010) & Mathur et al. (2010) $^{b}$ & Huber et al. (2012) & Ozel et al. (2013) $^{c}$ & Ozel et al. (2013) $^{d}$ \\
\hline$M / M_{\odot}$ & $1.31 \pm 0.06$ & $1.6 \pm 0.3$ & & $1.6 \pm 0.2$ & $1.3 \pm 0.2$ & $1.3 \pm 0.2$ \\
$t / \mathrm{Gyr}$ & $2.7 \pm 0.4$ & & & & $2.1 \pm 0.2$ & $2.3 \pm 0.3$ \\
$R / R_{\odot}$ & $1.60 \pm 0.03$ & $1.7 \pm 0.2$ & $1.61 \pm 0.03$ & $1.73 \pm 0.08$ & $1.6 \pm 0.1$ & $1.6 \pm 0.1$ \\
\hline
\end{tabular}

Notes. ${ }^{(a)}$ Scaling relations. ${ }^{(b)}$ Radius Extractor (Creevey et al. 2013). ${ }^{(c)}$ GN93. ${ }^{(d)}$ AGS05.

Table 4. Parameters for HD 175272 derived by Ozel et al. (2013).

\begin{tabular}{lcccc}
\hline \hline \multirow{2}{*}{ Mixture } & \multicolumn{2}{c}{ GN93 } & \multicolumn{2}{c}{ AGS05 } \\
$\Delta v$ & scaling & adiabatic & scaling & adiabatic \\
& rel. & freq. & rel. & freq. \\
\hline$M / M_{\odot}$ & $1.32 \pm 0.09$ & $1.3 \pm 0.4$ & $1.32 \pm 0.09$ & $1.3 \pm 0.6$ \\
$t / \mathrm{Gyr}$ & $1.6 \pm 0.3$ & $1.6 \pm 0.6$ & $1.8 \pm 0.2$ & $1.7 \pm 0.1$ \\
$R / R_{\odot}$ & $1.63 \pm 0.04$ & 1.62 & $1.63 \pm 0.04$ & 1.65 \\
\hline
\end{tabular}

are at best marginally consistent, with the radius being tightly constrained by the small uncertainty in the large frequency separation. It remains unclear why the discrepancy is so significant, although we found that the discrepancy is eliminated when only $T_{\text {eff }},[\mathrm{Fe} / \mathrm{H}]$ and $\Delta v$ are used as observational constraints (see Sect. 4 and Fig. 1).

Although the model results are mutually consistent, the values of $v_{\max }$ returned from the grid-based modelling are all systematically lower than the reported observed value. We have checked the literature for additional values for $v_{\max }$. For HD 181420 the following results are mentioned: $1574 \pm 13 \mu \mathrm{Hz}$ (Huber et al. 2012) and 1536.26 $\pm 0.42 \mu \mathrm{Hz}$ (Mathur et al. 2010). These values are intermediate between the observational value quoted by Ozel et al. (2013) and the results from our grid-based modelling.

\section{Discussion}

HD 181420 has been extensively studied with several techniques. Bruntt (2009) performed a detailed spectroscopic analysis of the star followed by an in-depth seismic analysis by Barban et al. (2009). Huber et al. (2012) obtained an independent radius measurement using interferometry. This star was further studied by Mathur et al. (2010) and Ozel et al. (2013). The parameters reported in these works are listed in Table 3. The masses and radii are roughly consistent with our results. The ages, where given, are slightly (and not always significantly) larger. We note that these results all correspond to lower masses. To achieve the same radius (which is strongly constrained by asteroseismology), lower-mass stars must be older, so we expect a correlation between the ages and masses. Additionally, the results obtained by Ozel et al. (2013) are derived using $\Delta v, T_{\text {eff }}$, and the luminosity $L$, while in the current analysis luminosity was not constrained, which could also influence the determined mass and age.

Being a lower signal-to-noise target, HD 175272 has been less extensively studied. Ozel et al. (2013) modelled HD 175272 using a differential analysis and their results are listed in Table 4. These are based on two solar mixtures (Grevesse \& Noels 1993, GN93; Asplund et al. 2005, AGS05) and two calculations of the large separation: either from the scaling relations or from a direct calculation of the adiabatic oscillation frequencies. Again, our results are broadly consistent. However, the uncertainties quoted by Ozel et al. (2013) predict that HD 175272 could have a subsolar mass, whereas its effective temperature is about $1000 \mathrm{~K}$ hotter than the Sun's. Based purely on the effective temperature of $6675 \pm 120 \mathrm{~K}$, and given that metallicity of HD 175272 is roughly solar, we would expect subsolar masses to be excluded at around the 5-sigma level. The uncertainties in our grid-based modelling are significantly smaller, and do not predict such unrealistic values. Thus, our results demonstrate that grid-based modelling can provide consistent and realistic stellar parameters even given only modest spectroscopic and asteroseismic constraints.

One may argue that the results presented here are only for the input physics implemented in the BASTI and MESA models, and thus the uncertainties quoted in Table 2 do not include a contribution from uncertainties in the model physics. Chaplin et al. (2014) performed a similar grid-based modelling analysis of a large sample of dwarfs and subgiant stars using 6 different grid-based pipelines and 11 stellar evolution codes. These authors conclude that, for stars for which spectroscopic data are available, as is the case for HD 175272 and HD 181420, the median uncertainties are approximately $5.4 \%$ in mass, $2.2 \%$ in radius and $57 \%$ of their sample have ages with uncertainties less than 1 Gyr. These values are of the same order as the values quoted in Table 2 and would not imply unphysically large uncertainties. We note here that only internal error bars are shown in Fig. 1 as literature values only mention internal uncertainties.

We now return to the discrepancy between the observed and modelled values of $v_{\max }$ (see also Ozel et al. 2013). For both stars all of our fits correspond to $v_{\max } \lesssim 1500 \mu \mathrm{Hz}$, which is significantly lower than the observed values of $\sim 1600 \mu \mathrm{Hz}$. The modelled values for $\Delta v,[\mathrm{Fe} / \mathrm{H}]$ and $T_{\text {eff }}$ are consistent with the observed values, given the uncertainties and restrictions from the model grid. In particular, both grids are relatively coarse in metallicity and the values returned are dominated by the closest possibilities compared to the observed values, with the uncertainties reflecting the width of the bins of either the likelihood distribution (LD) or the cumulative distribution function (PySEEK).

In addition, we found that by fitting the MESA models with the asymptotic large separations to the three constraints on $T_{\text {eff }}$, $[\mathrm{Fe} / \mathrm{H}]$ and $\Delta v$ (and not $v_{\max }$ ), the discrepancies with the results from the scaling relations are completely eliminated. The results of this fit are given in Table 5. For both HD 181420 and HD 175272, the model parameters become much more consistent with the other results. The values of $v_{\max }$ become more discrepant, but all other fitted parameters move towards either the observed values or the values from the other models. We conclude that $v_{\max }$ is the discrepant observational constraint in HD 181420 and HD 175272.

\section{Conclusions}

We have successfully used grid-based modelling to derive consistent and realistic stellar parameters for both the high signalto-noise target HD 181420 and the seismically similar star HD 175272. Our analysis employed two model-fitting methods and two sets of stellar models. For HD 181420 we derive values 
Table 5. Results for grid-based modelling of HD 181420 with only $\Delta v$, $T_{\text {eff }}$, and $[\mathrm{Fe} / \mathrm{H}]$ as observational constraints.

\begin{tabular}{lcc}
\hline \hline Model grid & $\begin{array}{c}\text { HD } 181420 \\
\text { MESA }^{a}\end{array}$ & $\begin{array}{c}\text { HD 175272 } \\
\text { MESA }^{a}\end{array}$ \\
method & PySEEK & PySEEK $^{+0.02}$ \\
\hline$M / M_{\odot}$ & $1.42_{-0.12}^{+0.2}$ & $1.47_{-0.04}^{+0.06}$ \\
$t / \mathrm{Gyr}$ & $2.0_{-0.2}^{+0.6}$ & $1.6_{-0.3}^{+0.4}$ \\
$R / R_{\odot}$ & $1.710_{-0.057}^{+0.004}$ & $1.71_{-0.01}^{+0.02}$ \\
$\Delta v / \mu \mathrm{Hz}$ & $75.20_{-0.01}^{+0.06}$ & $74.9_{-0.4}^{+0.4}$ \\
$\nu_{\max } / \mu \mathrm{Hz}$ & $1418_{-48}^{+7}$ & $1431_{-23}^{+28}$ \\
$T_{\text {eff }} / \mathrm{K}$ & $6582_{-136}^{+72}$ & $6666_{-113}^{+125}$ \\
{$[\mathrm{Fe} / \mathrm{H}]$} & $0.03_{-0.13}^{+0.11}$ & $0.08_{-0.11}^{+0.01}$ \\
\hline
\end{tabular}

Notes. ${ }^{(a)} \Delta v$ from asymptotic large frequency spacing $\left(\Delta v_{\text {as }}\right.$, see Eq. (3)).

in the ranges of $1.30-1.45 M_{\odot}, 1.59-1.714 R_{\odot}$ and $1.7-2.6 \mathrm{Gyr}$ in mass, radius, and age, respectively. In the case of HD 175272, we derive smaller uncertainties than the differential analysis by Ozel et al. (2013), providing results in a physically expected regime, i.e., our results lie in the ranges $1.40-1.57 M_{\odot}, 1.62-1.73 R_{\odot}$ and 1.27-1.64 Gyr for the mass, radius, and age, respectively. This demonstrates that grid-based modelling is useful even for low signal-to-noise targets. We also note that the modelled values of $\Delta v, T_{\text {eff }}$ and $[\mathrm{Fe} / \mathrm{H}]$ are consistent with the observed values given by Ozel et al. (2013), but the frequencies of maximum oscillation power $v_{\max }$ are significantly smaller in both stars. In one modelling case, we found that omitting $v_{\max }$ greatly improves the consistency of the models with the other observables. We conclude that these observed values of $v_{\max }$ are not consistent with the stellar models used here.

Acknowledgements. The authors would like to thank Jesper Schou, Hannah Schunker and Tim White for their comments on the manuscript. WHB acknowledges research funding by Deutsche Forschungsgemeinschaft (DFG) under grant SFB 963/1 "Astrophysical flow instabilities and turbulence" (Project A18). The research leading to the presented results has received funding from the European Research Council under the European Community's Seventh Framework Programme (FP7/2007-2013) / ERC grant agreement No. 338251 (StellarAges).

\section{References}

Angulo, C., Arnould, M., Rayet, M., et al. 1999, Nucl. Phys. A, 656, 3 Asplund, M., Grevesse, N., \& Sauval, A. J. 2005, in Cosmic Abundances as Records of Stellar Evolution and Nucleosynthesis, eds. T. G. Barnes, III, \& F. N. Bash, ASP Conf. Ser., 336, 25
Auvergne, M., Bodin, P., Boisnard, L., et al. 2009, A\&A, 506, 411

Baglin, A., Auvergne, M., Barge, P., et al. 2006, in ESA SP 1306, eds. M. Fridlund, A. Baglin, J. Lochard, \& L. Conroy, 33

Barban, C., Deheuvels, S., Baudin, F., et al. 2009, A\&A, 506, 51

Barclay, T., Rowe, J. F., Lissauer, J. J., et al. 2013, Nature, 494, 452

Basu, S., Chaplin, W. J., \& Elsworth, Y. 2010, ApJ, 710, 1596

Beck, P. G., Bedding, T. R., Mosser, B., et al. 2011, Science, 332, 205

Beck, P. G., Montalban, J., Kallinger, T., et al. 2012, Nature, 481, 55

Bedding, T. R., Mosser, B., Huber, D., et al. 2011, Nature, 471, 608

Böhm-Vitense, E. 1958, Z. Astrophys., 46, 108

Borucki, W., Koch, D., Basri, G., et al. 2008, in IAU Symp. 249, eds. Y.-S. Sun, S. Ferraz-Mello, \& J.-L. Zhou, 17

Borucki, W. J., Koch, D., Basri, G., et al. 2010, Science, 327, 977

Bruntt, H. 2009, A\&A, 506, 235

Cassisi, S., \& Salaris, M. 1997, MNRAS, 285, 593

Caughlan, G. R., \& Fowler, W. A. 1988, At. Data Nucl. Data Tables, 40, 283

Chaplin, W. J., Sanchis-Ojeda, R., Campante, T. L., et al. 2013, ApJ, 766, 101

Chaplin, W. J., Basu, S., Huber, D., et al. 2014, ApJS, 210, 1

Cox, J. P. 1968, Principles of stellar structure - Vol.1: Physical principles; Vol.2: Applications to stars (New York: Gordon and Breach)

Creevey, O. L., Thévenin, F., Basu, S., et al. 2013, MNRAS, 431, 2419

Deheuvels, S., García, R. A., Chaplin, W. J., et al. 2012, ApJ, 756, 19

Ferguson, J. W., Alexander, D. R., Allard, F., et al. 2005, ApJ, 623, 585

Gai, N., Basu, S., Chaplin, W. J., \& Elsworth, Y. 2011, ApJ, 730, 63

Grevesse, N., \& Noels, A. 1993, in Origin and Evolution of the Elements, eds. N. Prantzos, E. Vangioni-Flam, \& M. Casse, 15

Grevesse, N., \& Sauval, A. J. 1998, Space Sci. Rev., 85, 161

Hekker, S., Elsworth, Y., Basu, S., et al. 2013a, MNRAS, 434, 1668

Hekker, S., Elsworth, Y., Mosser, B., et al. 2013b, A\&A, 556, A59

Henyey, L., Vardya, M. S., \& Bodenheimer, P. 1965, ApJ, 142, 841

Huber, D., Bedding, T. R., Stello, D., et al. 2011, ApJ, 743, 143

Huber, D., Ireland, M. J., Bedding, T. R., et al. 2012, ApJ, 760, 32

Huber, D., Carter, J. A., Barbieri, M., et al. 2013, Science, 342, 331

Iglesias, C. A., \& Rogers, F. J. 1996, ApJ, 464, 943

Irwin, A. W. 2012, FreeEOS: Equation of State for stellar interiors calculations, astrophysics Source Code Library, ascl: 1211.002

Kjeldsen, H., \& Bedding, T. R. 1995, A\&A, 293, 87

Krishna Swamy, K. S. 1966, ApJ, 145, 174

Kunz, R., Fey, M., Jaeger, M., et al. 2002, ApJ, 567, 643

Mathur, S., García, R. A., Régulo, C., et al. 2010, A\&A, 511, A46

Mazumdar, A., Michel, E., Antia, H. M., \& Deheuvels, S. 2012, A\&A, 540, A31

Mazumdar, A., Monteiro, M. J. P. F. G., Ballot, J., et al. 2014, ApJ, 782, 18

Miglio, A., Montalbán, J., Carrier, F., et al. 2010, A\&A, 520, L6

Miglio, A., Brogaard, K., Stello, D., et al. 2012, MNRAS, 419, 2077

Mosser, B., Barban, C., Montalbán, J., et al. 2011, A\&A, 532, A86

Mosser, B., Goupil, M. J., Belkacem, K., et al. 2012, A\&A, 548, A10

Mosser, B., Michel, E., Belkacem, K., et al. 2013, A\&A, 550, A126

Ozel, N., Mosser, B., Dupret, M. A., et al. 2013, A\&A, 558, A79

Paxton, B., Bildsten, L., Dotter, A., et al. 2011, ApJS, 192, 3

Paxton, B., Cantiello, M., Arras, P., et al. 2013, ApJS, 208, 4

Pietrinferni, A., Cassisi, S., Salaris, M., \& Castelli, F. 2004, ApJ, 612, 168

Quirion, P.-O., Christensen-Dalsgaard, J., \& Arentoft, T. 2010, ApJ, 725, 2176

Reimers, D. 1975, Mem. Soc. Roy. Sci. de Liege, 8, 369

Salaris, M., \& Cassisi, S. 1998, MNRAS, 298, 166

Silva Aguirre, V., Casagrande, L., Basu, S., et al. 2012, ApJ, 757, 99

White, T. R., Bedding, T. R., Stello, D., et al. 2011, ApJ, 743, 161 